\title{
Formación ciudadana y construcción de comunidad: investigación acción colaborativa entre escuela y universidad
}

\author{
Citizenship education and community building: \\ University-School collaborative action-research
}

\begin{abstract}
Alicia Zamorano-Vargas ${ }^{\mathrm{a}}$ \& María Eugenia Hernández ${ }^{\mathrm{b}}$
aUniversidad de Chile, Santiago, Chile. $\square$ alicia.zamorano@uchile.cl [orcid.org/0000-0002-5821-180X]

bUniversidad de Chile, Santiago, Chile. me.hernandez@uchile.cl [orcid.org/0000-0002-2906-4345]
\end{abstract}

\section{RESUMEN}

La Formación Ciudadana puede ser entendida como un espacio ideal para la educación de ciudadanos que valoren la diversidad cultural, la democracia y la justicia social. En este artículo se expondrá las conclusiones respecto un proceso de investigación-acción colaborativa que se realizó en un centro educativo de la ciudad de Santiago de Chile y que tuvo entre sus objetivos desarrollar aprendizajes para la formación ciudadana en contextos de diversidad cultural. Para realizar esta investigación se contó con la participación de estudiantes de Enseñanza Básica. Como conclusiones podemos señalar la trascendencia del trabajo colaborativo entre el centro educativo y la universidad; el potencial de la I-A-C en el trabajo realizado con niños, niñas y adolescentes y, finalmente, una reflexión sobre el trabajo realizado con el centro respecto a conceptos de ciudadanía y el aporte que la investigación entre el centro y la universidad entrega a la formación inicial de docente.

\section{PALABRAS CLAVE: Formación Ciudadana, participación, investigación-acción- colaborativa.}

\section{ABSTRACT}

Citizenship Education can be understood as an ideal space for educating citizens who value cultural diversity, democracy and social justice. This article reports findings from a collaborative action-research process implemented in a school in the city of Santiago de Chile, which goals were to develop learning for citizenship education in contexts of cultural diversity. Elementary School students participated in the project. We conclude by signaling the importance of collaborative work between the school and the university; the potential of 


\section{A. Zamorano-Vargas \& M. E. HernándeZ}

collaborative action-research in working with school children and adolescents, and reflecting on the concept of citizenship and the contribution that both the school and the university makes to pre-service teacher education.

KEYWORD: Citizenship Education, participation, collaborative research.

\section{FORMACIÓN CIUDADANA Y ESCUELA}

La construcción de la ciudadanía a través de la escuela ha sido una constante preocupación de los Estados. En el presente, los distintos fenómenos sociales y políticos que afectan al mundo, que se manifiestan en cuestiones como la desafección política, la individualización, la desigualdad y la cada vez más constante manifestación de discursos de tipo xenófobos y excluyentes, entre otros, han provocado preocupación en los organismos públicos internacionales y nacionales sobre los conocimientos políticos de las y los jóvenes, lo que ha dado fuerza a una serie de políticas que potencian la agenda de la formación ciudadana en las escuelas (Bolívar, 2007). Frente a la tradicional construcción de una ciudadanía vinculada a los discursos nacionales hegemónicos y homogeneizantes, en el siglo XXI se plantea el desarrollo de una Formación Ciudadana que se reconfigure para colocar acento en la construcción de un concepto más amplio, que haga visible la pluralidad de identidades, la diversidad y la alteridad como características de la democracia actual (Pagès \& Santisteban, 2010).

En el caso de Chile, desde el retorno a la democracia hasta el presente, la Formación Ciudadana ha sido un tema central de las políticas educativas, lo que se ha materializado en cambios en el currículum, en las políticas evaluativas y en las leyes y normativas educacionales. Uno de los principales resultados de este proceso se concretó el año 2016 cuando se promulgó la Ley 20.911, que creó el 'Plan de Formación Ciudadana para los Establecimientos Educacionales'. Esta ley busca desarrollar una cultura democrática y ética en la escuela, a través de la formación de los y las estudiantes como ciudadanas y ciudadanos con virtudes cívicas, que participen en los temas de interés público, conocedores de la institucionalidad política y comprometidos con los Derechos Humanos.

Pero la ciudadanía y la educación ciudadana no son conceptos estáticos sino construcciones históricas y sociales que han conllevado a través del tiempo distintos significados y prácticas (Cortina, 2017; Bolívar, 2007). De acuerdo con Bolívar (2007), se puede distinguir tres tradiciones sobre la ciudadanía: liberalismo, comunitarismo y republicanismo cívico. La tradición del liberalismo representa una concepción individualista en el ejercicio de los derechos, donde el Estado intenciona un rol de los ciudadanos más bien pasivo y con escasos deberes en relación a lo común. Además, la formación de la ciudadanía debe tener una posición neutral, al menos en lo declarativo, frente a las diversas concepciones de vida que se presenten en la sociedad, lo que permite reforzar el valor de la tolerancia y el pluralismo. La tradición comunitarista, asocia la ciudadanía al sentido de pertenencia y desde ahí reivindica una visión multiculturalista, proponiendo un reconocimiento de la identidad cultural que puede abocar al desarrollo de una ciudadanía fragmentada o diferenciada. Desde esta tradición el ciudadano se define por un sentimiento de pertenencia a su comunidad, 
siendo la identidad la base para reivindicar derechos propios. La tradición del republicanismo cívico entiende la ciudadanía como una práctica participativa. Según esta tradición los temas políticos son de interés de todas y todos los integrantes de la comunidad y por lo tanto, la pertenencia a la sociedad se construye en torno a la participación en los asuntos de aquella. Desde esta perspectiva los derechos individuales están condicionados por los intereses de la comunidad y la identidad que se forma y une al grupo es una identidad cívica y no étnica como propusiera la tradición comunitarista (Bolívar, 2007; Giroux, 2004).

Las distintas tradiciones sobre ciudadanía también han tenido su expresión en la Formación Ciudadana. Al igual que en el currículum, de acuerdo con Giroux (2004), las prácticas escolares respecto de la construcción de la ciudadanía se pueden asociar a tres tipos de racionalidades que van desde una racionalidad técnica, que apuesta por la reproducción social, hasta una racionalidad emancipatoria que apuesta por la transformación de la realidad. En la escuela, la ciudadanía puede ser entendida como un concepto polisémico. Por una parte puede estar asociado a la idea de civilidad, referirse a tener conductas respetuosas con las reglas de la vida en común, corresponder con la idea de Educación Cívica, que significa aprender cómo funcionan las instituciones; o vincularse a una perspectiva que coloca el foco en el estudio razonado y crítico de los problemas sociales y desde ahí conlleva una Formación Ciudadana con énfasis en el desarrollo de la participación ciudadana (Heimberg, 2010).

Esta última forma de concebir la Formación Ciudadana en la escuela es la que aparece como representativa de los proyectos que en esta área se han levantado en Chile. Esta perspectiva ha sido denominada como concepción máxima de la ciudadanía y supone conocimientos sobre el sistema democrático y la institucionalidad política pero, además, el desarrollo de competencias por parte de los y las estudiantes (Cox, Jaramillo \& Reimers, 2005), de habilidades, valores y disposiciones que les permitan desenvolverse como ciudadanos plenos en una sociedad democrática y en la vida cívica en sus distintos niveles (Magendzo \& Gazmuri, 2018). El desarrollo de la ciudadanía en la escuela desde esta perspectiva implica el desafío de sostenerse sobre procesos que tienen como foco el entender la democracia como un concepto inacabado, donde los y las estudiantes se enfrenten al desarrollo de la discusión de lo controversial y socialmente relevante en la escuela (Hess, 2009; Hess \& McAvoy, 2015). Vale decir, que la escuela debe enfrentar el reto de construirse como un espacio democrático, que debe ser capaz de abordar las distintas problemáticas que se desarrollan en el mundo real de una forma significativa para las y los estudiantes. Entre estos desafíos se encuentra el atender a la diversidad y que la escuela se transforme en un espacio inclusivo (Bolívar, 2007; Heimberg, 2010; Magendzo \& Gazmuri, 2018).

\section{LA INVESTIGACIÓN CON LOS CENTROS EDUCATIVOS}

Comenzamos este trabajo desde la idea de que investigar en educación permite la creación de un cuerpo organizado de conocimientos científicos y que, además, será del interés de los educadores (Travers, 1986). Para el desarrollo de esta investigación nos centramos en un paradigma cualitativo, ya que nuestro interés radicó en comprender y explorar la conducta de los sujetos dentro del escenario educativo (Hernández, Fernández \& Baptista, 2014; Latorre, del Rincón \& Arnal, 2005). En la misma línea y siguiendo a Stake (2007) uno 


\section{A. Zamorano-Vargas \& M. E. HernándeZ}

de los intereses de la investigación cualitativa está en la "comprensión de las complejas relaciones entre todo lo que existe" (p. 42).

En particular, y según el interés de esta investigación, nos decidimos por el enfoque metodológico de la Investigación Acción. Si bien existen variadas aproximaciones sobre este tipo de investigación, hay varios autores que le atribuyen a John Dewey sus ideas fundacionales; esto, debido a su concepción sobre la Pedagogía, es decir, el carácter democrático de la Educación, el aprendizaje en la acción y la necesidad de la implicación de los docentes. El pensamiento crítico y reflexivo de Dewey junto a su concepción de democracia y participación están presentes (implícita o explícitamente) en los planteamientos de la investigación-acción, pero en definitiva quien logró consolidarlo fue Lewin en 1946, al afianzar la idea de que la investigación es investigación-acción, ya que así los avances teóricos y los cambios sociales pueden lograrse simultáneamente (Latorre et al. 2005)

Por la propia naturaleza de este tipo de investigación y la forma como se ha ido consolidando, los autores admiten que es difícil llegar a una conceptualización unívoca. Para Carr \& Kemmis (1988) "la I-A es una forma de indagación autorreflexiva que emprenden los participantes en situaciones sociales en orden a mejorar la racionalidad y la justicia de sus propias prácticas, su entendimiento de las mismas y las situaciones dentro de las cuales ellas tienen lugar" (p. 174); no obstante, en la actualidad la I-A se ha transformado en una familia de enfoques que tienen como características en común a) ser una perspectiva alternativa a la concepción positivista que defiende la unión entre investigador e investigado; b) ser un trabajo democrático, ya que se realiza desde una perspectiva comunitaria; c) se realiza de manera conjunta, para tomar decisiones que permita la creación de comunidades autocríticas con el fin de transformar el medio social.

Con el paso del tiempo y diversas investigaciones se han diferenciado como corrientes, la I-A del Profesor, I-A Participativa y la I-A Colaborativa (Rodríguez, Gil \& García, 1999; Latorre et al. 2005; Villalta \& Ortega, 2019)

La I-A del profesor, o Investigación-Acción en la escuela se basa en las ideas de Elliot (2005) quien indica que el objetivo fundamental de la investigación acción consiste en mejorar la práctica en vez de generar conocimientos. Busca perfeccionar la práctica mediante el desarrollo de las capacidades de discriminación y de juicio profesional en situaciones concretas y humanas, especificando que quien desarrolla la investigación es el profesor.

La I-A Participativa, plantea que se puede obtener conocimientos colectivos sobre una realidad social determinada. En este tipo de investigación se conjugan la investigación social, el trabajo educativo y la acción. El problema a estudiar surge desde la comunidad, y lo que se busca es su transformación con el fin de desarrollar mejoras en las interacciones de los sujetos implicados. Junto a ello, tiene por objetivo que la comunidad logre una toma de conciencia sobre sus propias habilidades y posibilidades de movilización.

En el caso de este trabajo nos centraremos en la Investigación Acción Colaborativa. La I-A-C, también denominada Cooperativa (Rodríguez et al.1999), que tiene como característica básica 'investigar en colaboración' o ‘co-investigar', donde un conjunto de personas investiga conjuntamente situaciones o problemas compartidos, relevando una relación horizontal y no jerarquizada entre sus actores. Esto significa que adopta "el principio de trabajar con, no trabajar sobre” lo que ocurre con los profesores y las escuelas (Latorre et al. 2005). 
Por estas razones nos decidimos por la I-A-C, ya que nos interesaba desarrollar un trabajo en conjunto con el centro educativo, que nos permitiera trabajar de manera asociada sobre un problema que la comunidad considerara importante y, desde ese punto de partida, que no solo tuviera como finalidad la reflexión académica, sino también significara desarrollo profesional para la comunidad de docentes que participaron, y así mejorar y comprender mejor su práctica, lo que se traduce en la mejora de los aprendizaje de los/las estudiantes y en su experiencia dentro de la escuela.

\section{METODOLOGÍA}

Esta investigación se realizó en un centro educativo de la ciudad de Santiago de Chile y para conocer e interpretar la realidad utilizamos el método de la Investigación-AcciónColaborativa.

A este centro educativo asisten niños, niñas y jóvenes desde los 4 hasta los 18 años. El índice de vulnerabilidad indicado por el Ministerio de Educación para el año 2018 fue de 68\% con un número creciente de matrícula de estudiantes extranjeros. Dadas estas características, las y los profesores del centro propusieron que trabajáramos en conjunto para mejorar los aprendizajes de los niños y niñas, intencionando acciones desde la convivencia escolar, es por eso que la investigación tuvo como objetivo desarrollar aprendizajes para la Formación Ciudadana en contextos de diversidad cultural, lo que nos orientó a involucrarnos en las acciones que se desarrollaban dentro del centro educativo, para comprender las experiencias y el punto de vista de los sujetos.

Siendo característico de la I-A-C, los/as investigadores/as fueron profesores y profesoras del centro educativo, profesoras de la universidad y estudiantes de la universidad que realizaban su práctica en el centro.

El primer planteamiento de esta investigación contemplaba el desarrollo de competencias profesionales en el equipo docente del establecimiento desde un enfoque de Educación intercultural. Para el desarrollo del estudio se utilizó el aprendizaje por proyectos, porque considera la inclusión de actividades que promueven 1) el planteamiento y resolución de problemas; 2) la promoción de actividades que permitan promover la comprensión para actuar; 3) actividades que fomenten el desarrollo de la racionalidad y la comunicación; 4) la relación de los procesos políticos y ciudadanos con la dimensión espacial y temporal; 5) la formación del pensamiento sobre el futuro (Santisteban, 2009).

En este sentido se planearon experiencias de aprendizaje que facilitaran la formación de ciudadanos que se inserten en una comunidad diversa, valorando las diferencias y reconociendo elementos que las diversas culturas tienen en común. El curso en que se aplicaría inicialmente dicho proyecto era Quinto Básico ya que este nivel presentaba la dificultad de ser un curso de transición entre la Educación Básica y Media y porque este grupo en particular manifestaba problemas de convivencia. La investigación se inició con entrevistas al jefe de la Unidad Técnico Pedagógica (UTP) donde se abordaron las características y necesidades del curso elegido, con la finalidad de estructurar el proyecto y presentarlo a los y las docentes de Quinto Básico. Nuestra propuesta consistía en partir desde sus percepciones sobre 


\section{A. Zamorano-Vargas \& M. E. HernándeZ}

interculturalidad, democracia y ciudadanía para abordarlas desde la realidad en el centro con la finalidad de producir reflexiones e innovaciones en las prácticas pedagógicas y en las dinámicas educativas (Villalón, 2012). En la primera de las cuatro reuniones realizadas con los docentes -luego de presentar el proyecto y comentarlo-, uno de ellos recalcó la importancia de generar un discurso común entre adultos sobre ciudadanía, interculturalidad y democracia para que luego se pudiera organizar en conjunto las actividades con los y las estudiantes y así evitar el que ellos tuvieran que adoptar concepciones traídas desde fuera, lo cual es consistente con el desarrollo de una I-A-C, donde se trabaja conjuntamente tanto en la toma de decisiones como la realización de las tareas, a diferencia con lo que ocurre con otros tipos de investigación donde los investigadores son agentes externos y expertos que llegan a la escuela a implementar mejoras. Luego de esto se organizaron sesiones de trabajo: en una primera instancia se aplicó un cuestionario y se realizaron dos grupos focales para indagar sobre las percepciones de los docentes sobre temas de interculturalidad, democracia y ciudadanía en la escuela (septiembre 2019); en una segunda oportunidad revisamos alcances teóricos sobre multiculturalidad, interculturalidad y transculturalidad y su vinculación con la democracia y ciudadanía (octubre 2019); finalmente se llegó a un concepto común sobre interculturalidad y su aporte a una escuela más democrática (octubre 2019).

El siguiente paso consistía en la determinación de los Objetivos de Aprendizaje que se trabajarían en conjunto con los y las profesoras de Quinto Básico, pero debido a la crisis social que comenzó el 18 de octubre de 2019, no se realizó como estaba previamente organizado. Este obstáculo dentro del proceso nos impidió desarrollar un trabajo con toda la comunidad escolar, incluidos los padres, madres y apoderados, lo cual fue una pérdida para la investigación ya que consideramos que su participación es fundamental para el logro de aprendizajes en niños y niñas y un agente relevante a la hora de trabajar con los conceptos de democracia, ciudadanía e interculturalidad que habíamos definido como conceptos claves para la convivencia entre las y los estudiantes del Quinto Año Básico.

Finalmente, al modificar la estrategia para el trabajo con las y los estudiantes, y en base a los talleres y los grupos focales, se propuso al equipo un taller de tres días nominado 'Construcción de comunidad desde la participación de niños, niñas y adolescentes'. Este taller se realizó durante la segunda semana de diciembre (primera semana de vacaciones de los y las estudiantes), con un grupo multinivel del centro. Durante cada día se trabajó con objetivos específicos: la finalidad del primer día fue reconocer que a pesar de nuestras diversas características y experiencias de vida, somos iguales en derechos y dignidad; en el segundo día se buscó reconocer qué es un conflicto, cómo se producen y qué podemos hacer para solucionarlos, junto con profundizar la idea de que los conflictos son parte de la vida cotidiana y que no son un problema; y el objetivo del tercer día fue reconocer que a partir de las experiencias, la convivencia y el tiempo compartido nos convertimos en comunidad, con una identidad propia, donde todos podemos aportar desde nuestras características personales.

En síntesis, se trabajó con tres docentes de Quinto Básico con quienes se realizaron cuatro reuniones, instancias donde se presentó el proyecto, se aplicó un cuestionario y construyó un marco conceptual a través de grupos focales y, a partir de esto, se realizó un taller de tres días $(9,10$ y 11 de diciembre) con seis niñas/os entre 9 y 13 años. 
A partir de las acciones mencionadas anteriormente, la siguiente tabla explicita las características principales de la I-A-C que se desarrollaron en el centro (Tabla 1). Vale decir, la definición en conjunto del problema de investigación (docentes y equipo de la universidad), la búsqueda en conjunto de las posibles soluciones y el desarrollo profesional tanto de docentes como de los investigadores externos. Cabe mencionar que si bien en la I-A-C, tanto los docentes como el equipo de la universidad se relacionan como co-investigadores, hemos decidido distinguir cada actor con la finalidad de facilitar la lectura de la Tabla 1.

Tabla 1. Características de la I-A-C y su correlación con las acciones en el centro.

\begin{tabular}{ll}
\hline Característica de la I-A-c & Acciones en el centro \\
\hline $\begin{array}{l}\text { Problema de investigación } \\
\text { mutuamente definidos }\end{array}$ & $\begin{array}{l}\text { Se establecieron reuniones para determinar algún problema relevante de la } \\
\text { comunidad y que desde la universidad pudiera apoyarse en su solución. }\end{array}$ \\
$\begin{array}{l}\text { Investigadores y profesores } \\
\text { buscan las posibles soluciones }\end{array}$ & $\begin{array}{l}\text { Se realizaron reuniones entre el equipo de profesores y el equipo desde la } \\
\text { universidad para consensuar posibles formas de trabajo. }\end{array}$ \\
& $\begin{array}{l}\text { Se elaboró en conjunto con los docentes una definición consensuada } \\
\text { sobre interculturalidad, se reflexionó sobre los conflictos de los y las }\end{array}$ \\
$\begin{array}{l}\text { Los profesores desarrollan } \\
\text { habilidades y conocimientos, } \\
\text { mientras los investigadores se }\end{array}$ & $\begin{array}{l}\text { estudiantes, la forma de enfrentarlos (adultos y niños) y su aporte a una } \\
\text { reeducan utilizando metodologías } \\
\text { de investigación }\end{array}$ \\
$\begin{array}{l}\text { Se organizaron encuestas y grupos focales con los y las docentes, } \\
\text { se sistematizó y analizó la información, se tomaron decisiones y se } \\
\text { reestructuraron las sesiones de acuerdo con los resultados que se } \\
\text { obtenía, recursivamente. }\end{array}$ \\
\hline
\end{tabular}

Fuente: Elaboración propia.

En cuanto al proceso de investigación que se desarrolló en el centro, el esquema de la Tabla 2 detalla momentos característicos de la I-A-C y su correlación con las acciones realizadas en el centro.

El modelo de investigación que utilizamos sigue las ideas propuestas por Lewin y ampliadas por Townsend (citado por Wood y Smith, 2018). Este modelo plantea un proceso circular y recursivo que comprende una serie de acciones de parte del equipo investigativo, las que son desarrolladas en el centro:

1. Fase de reconocimiento, se problematiza una situación o acción que requiere mejoras y se recopila la información para asegurar una caracterización adecuada y coherente con la realidad.

2. Reflexionar sobre el proceso: se identifican los elementos requeridos para el trabajo en común entre investigadores externos e internos.

3. Planificar para la acción: se determina un plan de acción para mejorar la situación inicial.

4. Implementa la acción: se desarrollan las acciones planificadas.

5. Reflexionar y evaluar el cambio: se reflexiona sobre las acciones realizadas y sus efectos en relación a la problemática y el contexto en que se realizan.

6. Pulir el enfoque: se toman nuevas decisiones a partir de la reflexión anterior para elaborar un nuevo ciclo de acción. 


\section{A. Zamorano-Vargas \& M. E. HernándeZ}

Tabla 2. Momentos de la I-A-C y su correlación con las acciones en el centro educativo.

\begin{tabular}{|c|c|}
\hline Momentos de la I-A-C & Acciones en el centro \\
\hline $\begin{array}{l}\text { Partir de un problema concreto de los profesores e } \\
\text { intentar sistematizar de alguna forma la dificultad }\end{array}$ & $\begin{array}{l}\text { El equipo determinó que existía un problema } \\
\text { en el aprendizaje en el Quinto Año Básico. Este } \\
\text { curso estaba conformado por un porcentaje de } \\
\text { estudiantes inmigrantes y presentaba problemas de } \\
\text { convivencia. }\end{array}$ \\
\hline $\begin{array}{l}\text { Se acumula evidencia empírica sobre el problema } \\
\text { estudiado }\end{array}$ & Se realizaron talleres y grupos focales. \\
\hline $\begin{array}{l}\text { La interpretación de los resultados permite enriquecer la } \\
\text { visión del problema }\end{array}$ & $\begin{array}{l}\text { El equipo de trabajo realizó una sistematización de } \\
\text { los resultados y se lograron definiciones comunes. }\end{array}$ \\
\hline Puede obtenerse cierta estructura de generalización & $\begin{array}{l}\text { A partir de la generalización y a pesar de las } \\
\text { dificultades del contexto se logró desarrollar un } \\
\text { taller de tres días con estudiantes. }\end{array}$ \\
\hline
\end{tabular}

Fuente: Elaboración propia.

En el centro educativo estas fases se tradujeron en las siguientes acciones:

1) Fase de reconocimiento: Esta fase fue utilizada para recopilar información sobre el problema detectado y determinado para trabajar durante la investigación, con la finalidad de verificar que el problema fue caracterizado adecuadamente y que la intervención sea lo más significativa posible. En esta etapa se realizaron los talleres y grupos focales con los profesores.

2) Reflexionar sobre el proceso: tanto los investigadores externos (universidad) como los internos (profesores del centro) determinaron que era necesario desarrollar un lenguaje común para la comunicación eficaz.

3) Planificar para la acción: en ese contexto se planifica la intervención a través de la enseñanza basada en proyectos que tendría como foco un objetivo de aprendizaje de quinto básico y que pudiera ser trabajado entre asignaturas. Esta planificación primera se reorganizó por las dificultades contextuales, pero tuvo en cuenta las fases anteriores. Estas acciones decantaron en la planificación de un taller realizado durante tres días, al que fueron invitados estudiantes de distintos niveles del centro.

4) Implementar la acción: Se realizó un taller de tres días que se detallan a continuación.

Día 1: ¿Somos iguales o distintos?

Objetivo:

Reconocer que, a pesar de nuestras diversas características y experiencias de vida, somos iguales en derechos y dignidad.

Actividades:

1. Dinámica de la línea al centro: el grupo de estudiantes se divide en dos, cada grupo se ubica a un lado dela línea divisoria. Dos monitores hacen preguntas para 
conocernos. Cada vez que una respuesta a la pregunta sea positiva, la persona se acerca a la línea y luego vuelve a su lugar.

2. ¿Cómo nos presentamos a los otros? En la sala habrá disposición de materiales (témperas, arcilla, plastilina, cartulinas) para que cada uno/a escoja una manera de caracterizarse y darse a conocer a los demás.

3. Plenario y cierre:

¿Por qué me representé de esta forma?

¿Qué quiero que los demás conozcan de mí?

¿Qué expectativas tengo de estos días del taller?

\section{Día 2: No estamos solos}

Objetivo:

Reconocer qué son los conflictos, cuándo emergen, qué podemos hacer para solucionarlos.

Profundizar en la idea de que los conflictos son parte de la vida cotidiana y que no son un problema.

\section{Actividades:}

1) Preguntas abiertas para intencionar el diálogo entre los y las participantes:

¿Me he sentido solo?

¿Cuándo? ¿Por qué?

¿Estar con alguien quiere decir necesariamente que esté acompañado?

2) Cada integrante del taller elige un conflicto de su vida que le ha sido significativo y lo representa a través de una escultura en arcilla. Durante la actividad conversamos sobre lo que es un conflicto, qué nos pasa cuando tenemos un problema con otra/o u otras/os, qué elementos nos cuesta de ellos, cuáles acciones nos han resultado y cuáles no.

3) Desafío: ¿Quieren realizar una actividad para compartir una merienda?

Decidir entre pastel y cosas saladas.

Análisis sobre la discusión desarrollada para tomar la decisión, a partir de interrogantes directrices sobre qué sentimos, qué elementos dificultan la toma de decisión y qué elementos ayudan a llegar a un acuerdo.

\section{Día 3: Somos comunidad}

Objetivo: Reconocer que, a partir de las experiencias, la convivencia y el tiempo compartido nos convertimos en comunidad, con identidad propia y en la que todos podemos aportar desde nuestras características personales.

Actividades:

1) Silla Musical ${ }^{1}$

1 En este caso la finalidad del juego fue inverso al tradicional. El desafío estuvo en lograr que todos los participantes pudiéramos organizarnos para poder sentarnos en una cantidad menor de sillas, terminando el equipo entero sentados en solo una. 


\title{
A. Zamorano-Vargas \& M. E. HernándeZ
}

2) ¿Qué es una comunidad? Construcción de base común; Derechos y deberes; Características de esta comunidad

3) "El colegio que soñamos" ¿Qué nos gusta del colegio, qué quisiéramos mejorar?

Elegir una meta pequeña, concreta y a partir de ella plantear una estrategia para conseguirla.

\author{
LA INVESTIGACIÓN ACCIÓN COLABORATIVA COMO \\ HERRAMIENTA GENERADORA DE APRENDIZAJES PARA LA \\ FORMACIÓN CIUDADANA EN CONTEXTOS DE DIVERSIDAD CULTURAL
}

La pregunta central que motiva esta I-A-C tiene relación con la interrogante sobre cómo co-construir estrategias de mejora en los aprendizajes para la formación ciudadana en niñas, niños y adolescentes, incluyendo sus diversas culturas, las que se tensionan, conjugan y conviven en un centro educativo altamente vulnerable.

Como explicamos en apartados anteriores, en coherencia con I-A-C, adecuamos el trabajo inicial que nos habíamos propuesto debido al contexto, recogiendo el trabajo realizado con los y las docentes procurando responder tanto a las necesidades del centro como al momento social que vivíamos. Si bien planificamos un taller con objetivos y actividades para cada día, estas últimas fueron replanteándose a partir de las inquietudes de niñas, niños y adolescentes (NNA), implementando recursivamente las diversas etapas de la I-A-C, para definir cómo alcanzaríamos los objetivos de cada día.

Este taller buscó que a partir del trabajo de NNA, se reconocieran como parte de una comunidad y valoraran tanto las diferencias como los puntos de encuentro entre ellos/as. Consideramos especialmente relevante trabajar el concepto de comunidad por el momento de incertidumbre que se estaba viviendo en Santiago y por ser un eje central del que deriva la buena convivencia, la identidad de grupo, el reconocimiento de la diversidad y la participación democrática. En este último aspecto, consideramos relevante informar a NNA la forma en que se trabajaría cada objetivo, con la finalidad de hacer ajustes a partir de sus propias voces e intereses.

El concepto de diversidad fue trabajado mediante diferentes actividades (dinámicas, juegos, trabajo con material concreto, elaboración de esculturas, síntesis a través pequeñas frases) que permitieron dialogar y compartir las percepciones que NNA tienen sobre la igualdad y la diferencia entre seres humanos. Niños, niñas y adolescentes vieron en esta diversidad de edades y orígenes una posibilidad de enriquecimiento mutuo. "Aquí aprendemos todos de todos" afirma uno de los integrantes del taller, cuando una de las adolescentes manifiesta que le llama la atención que el chico venezolano tenga reflexiones que a ella le parecen de "adultos", a pesar de la poca edad; o la inclusión de vocablos venezolanos dentro de las conversaciones que se daban mientras se realizaban las actividades, integrándolos a los propios repertorios discursivos.

Esta misma diversidad permitió, según las propias conclusiones del grupo, que tuvieran mayores opciones para solucionar conflictos y desafíos a los que fueron sometidos durante los días del taller: "la diferencia ayuda" y "conociéndonos descubrimos varias formas de 
lograr la finalidad" fueron expresiones que surgieron cuando analizaron la forma en que superaron el desafío de elegir entre dos alternativas para organizar la convivencia final2: al darles dos opciones entre las que debían elegir, tras la discusión el grupo llegó a una tercera alternativa, al constatar que ninguna de las dos opciones proporcionadas por los/ las adultos/as satisfacía a todos/as. Esta experiencia de llegar a una alternativa diferente de la proporcionada por los/las adultos/as habla también de su capacidad de desarrollar pensamiento divergente a partir de pequeños desafíos lo que incide en su implicación y participación en las actividades cuando se les da la oportunidad de manifestar sus propias ideas para mejorar y adaptar a su propio contexto las actividades.

Para abordar la convivencia (a partir de las percepciones manifestadas por los docentes), se trabajó como eje central el conflicto a partir de la narración de experiencias vividas por las y los estudiantes. Esta actividad se realizó con arcilla a petición explícita del grupo durante el día anterior, por lo que la propuesta fue comunicar a través de una escultura conflictos significativos de su vida. Durante el desarrollo de la actividad, cada uno/una fue contando alguna experiencia de conflicto relevante para sí mismo/a. Los conflictos narrados fueron de distinta naturaleza: desde experiencias de violencia vividas a partir de revueltas sociales (en Chile y Venezuela), dificultades para romper con estereotipos de género para una adolescente que le gustaba jugar al fútbol (considerado aun como una actividad poco 'femenina'), hasta problemas de convivencia que surgen en la vida cotidiana en la escuela. A pesar de las diferencias de edad entre los y las participantes, los conflictos expuestos fueron interesantes y significativos para todos/as el grupo escuchó con respeto, dieron alternativas de solución y manifestaron sus puntos de vista sobre lo conversado.

El papel de los adultos tanto en estas actividades fue secundario. Observamos que ese espacio de respeto, atención y apertura hacia las voces de NNA fue significativo para el grupo. Este espacio de autonomía no derivó en un 'desorden' de la actividad, sino que constituyó la apertura de un espacio de confianza, donde 'el poder' se comparte entre niños/ as, adolescentes y adultos. Al constatar que tanto niños y niñas como adolescentes eran valorados/as y validados/as en sus aportes, se incrementó la colaboración generando una comunidad crítica que reflexionó sobre diversas situaciones.

A partir de ello, esta nueva comunidad concluyó, que a pesar de que los conflictos son situaciones problemáticas que producen sentimientos negativos como tristeza o enojo, estos se pueden superar a través de la empatía, el respeto por la opinión de los demás y la búsqueda común de acuerdos.

Esta diversidad de culturas, la participación democrática a través de la toma de decisiones, el replanteamiento de las mismas en respuesta al contexto o intereses de las y los NNA presentes en el taller y la acogida de decisiones que escapan a las alternativas propuestas por los adultos, además de la concepción de la formación ciudadana -entendida como la convivencia dentro del grupo-, fomentaron la implicancia y permanencia dentro de un taller realizado en tiempos de vacaciones, con un grupo de adultos desconocidos y externos al colegio. Esto se evidencia en la regularidad y puntualidad en la asistencia de quienes participaron en el taller.

Véase actividades del segundo día del taller. 


\section{A. Zamorano-Vargas \& M. E. Hernández}

Vemos cómo a partir de la implementación de la I-A-C, se facilita que NNA se transformen en protagonistas de la propia construcción de sus aprendizajes, en este caso, sobre el construir comunidad. Es así como en las conclusiones del taller, ellos elaboraron las características que posee una comunidad, vale decir, aceptar las diferencias, ayudarnos entre todas y todos, conocernos, jugar juntos, estar a gusto, valorarnos.

\section{CONCLUSIONES}

Las conclusiones del presente artículo se presentarán tomando en cuenta tres ámbitos de la investigación acción realizada, a saber: el trabajo entre el centro educativo y la universidad; el trabajo realizado con los niños, niñas y adolescentes del centro y, finalmente, una reflexión del trabajo realizado con la escuela respecto al objetivo de desarrollar aprendizajes para la Formación Ciudadana en contextos de diversidad cultural, lo que incluye los conceptos de ciudadanía y el aporte de la investigación entre el centro y la universidad como insumo relevante para la formación inicial de profesores y profesoras.

En cuanto a los elementos que nos permiten hablar de un trabajo exitoso de I-A-C entre centros educativos y universidades, destacamos que el centro facilitó y propició el trabajo en conjunto entre los profesores y profesoras con la universidad, consensuando los días de trabajo entre los equipos. Si bien es complejo para un/a docente destinar tiempo para un trabajo que no está incluido en su jornada regular, los y las docente participantes organizaron su jornada laboral para realizar un trabajo en conjunto. Lo que perjudicó un mayor involucramiento de los docentes fueron situaciones ajenas al centro: la crisis social de octubre de 2019 y luego la pandemia de 2020.

Otro de los elementos que se consideran relevantes para realizar un proceso exitoso de I-A-C es la disposición de recursos para el desarrollo de la investigación. En este caso desde la universidad se dispuso de personal de apoyo a la investigación (estudiantes de pregrado) además de materiales para el desarrollo de las distintas sesiones de trabajo, lo que permitió desarrollar las sesiones de trabajo y el taller final.

En tercer lugar, es importante destacar las características y habilidades del equipo investigador. Cuando se analiza el avance de la investigación hasta antes de la crisis social, los profesores del centro fueron participantes clave para la elaboración de un estado de situación de los conceptos involucrados en el logro del objetivo (formación ciudadana, diversidad cultural) y que fueron fundamentales para la construcción del taller final realizado con los estudiantes. De la misma manera, el equipo de investigadores de la universidad logró adecuar las acciones propias de la I-A-C de manera que siempre se mantuviera el diálogo con la comunidad del centro y finalmente convocar a los niños y niñas y adolescentes.

En cuarto lugar, se debe considerar la claridad de los objetivos investigativos, el tiempo destinado al trabajo y las condiciones para establecer un trabajo conjunto entre el centro y la universidad. En este punto es importante relevar que los objetivos de la investigación fueron discutidos entre todos los participantes de la I-A-C, es decir el equipo del centro y el equipo de la universidad, lo que también contribuyó a establecer un calendario de trabajo que, si bien fue modificado, pudo desarrollarse para el cumplimiento de lo estipulado. Con 
respecto a las condiciones de trabajo, se debe destacar que la flexibilidad del equipo desde la universidad, la valoración del trabajo docente y la comprensión de la posible desconfianza hacia los 'expertos' externos que los profesores del centro podrían manifestar, permitió que pudiera desarrollarse confianza en los procesos y establecer un trabajo horizontal y colaborativo.

Respecto del aprendizaje de niños, niñas y adolescentes en las sesiones de trabajo realizadas, la I-A-C les dio oportunidades no solo de construir conocimientos, sino de aportar ideas desde su propia perspectiva para mejorar su experiencia escolar personal y de la comunidad. Este tipo de investigación nos permitió desplazar los límites del adultocentrismo en la toma de decisiones sobre qué y cómo enseñar y aprender para realzar y valorar las ideas de niños y niñas. Se pudo constatar que a través de la creatividad de los/las niños/ as se amplían las posibilidades de aprendizaje, se potencializan estas experiencias a través de la colaboración entre unos y otros, y aumenta el compromiso y adherencia a diversas actividades. Esto es relevante tanto para que los docentes de los centros educativos consideren e intencionen la participación de los niños, niñas y adolescentes en torno al desarrollo de comunidad y así también fomentar una ciudadanía activa, de manera de incluirlos efectivamente en su propia formación.

Ahora, sobre el involucramiento del centro educativo en el proyecto de investigación, destacamos que la Formación Ciudadana ha logrado posicionarse en la agenda educativa a partir del año 2016 con la promulgación del 'Plan de Formación Ciudadana para los Establecimientos Educacionales', por lo tanto, en un desafío reciente para las escuelas. En el caso de esta I-A-C, el trabajo del taller con niñas, niños y adolescentes permitió definir habilidades y actitudes para una convivencia cívica más armónica dentro de la comunidad.

Un elemento a destacar es que este proyecto de investigación entre universidad y escuela permitió una conexión estrecha entre dos instituciones que tienen fines similares, pero que no siempre desarrollan un diálogo. Es a partir de un trabajo conjunto donde se logra identificar, conocer y reflexionar sobre las necesidades concretas del centro educativo, ya que generalmente las necesidades se determinan desde lugares que no son la escuela. Finalmente, destacamos que experiencias investigativas conjuntas permiten a los formadores de formadores adquirir herramientas y conocimientos del contexto escolar, las que pueden ser transferidas directamente a la formación inicial docente y así contribuir significativamente a nuestro sistema educativo.

\section{REFERENCIAS}

Bolívar, A. (2007). Educación para la Ciudadanía. Algo más que una asignatura. Barcelona: Graó.

Carr, W., \& Kemmis, S. (1988). Teoría crítica de la enseñanza. La investigación-acción en la formación del profesorado. Barcelona: Ediciones Martínez Roca.

Cortina, A. (2017). Ciudadanos del mundo. Hacia una teoría de la ciudadanía. (3 ${ }^{\text {a }}$ Ed.). Madrid: Alianza Editorial.

Cox, C., Jaramillo, R., \& Reimers, F. (2005). Educar para la Ciudadanía y la Democracia en las 


\section{A. Zamorano-Vargas \& M. E. HernándeZ}

Américas: Una Agenda para la Acción. Banco Interamericano de Desarrollo: Washington D.C.

Elliot, J. (2005). El cambio educativo desde la investigación-acción (4a Ed.). Madrid: Morata. Giroux, H. (2004). Teoría y resistencia en educación (6 $6^{\mathrm{a}}$ Ed.). México D.F.: Siglo XXI Editores. Heimberg, C. (2010). ¿Cómo puede orientarse la educación para la ciudadanía hacia la libertad, la responsabilidad y la capacidad de discernimiento de las nuevas generaciones? Íber, $64,48-57$.

Hernández, R., Fernández, C., \& Baptista, P. (2014). Metodología de la investigación (6 ${ }^{\mathrm{a}}$ Ed.). México D.F.: McGraw-Hill.

Hess, D. (2009). Controversy in the classroom. The democratic power of discussion. New York: Taylor \& Francis.

Hess, D., \& McAvoy, P. (2015). The political classroom. Evidence and Ethics in Democratic Education. New York: Taylor \& Francis.

Latorre, A., del Rincón, D., \& Arnal, J. (2003). Bases metodológicas de la investigación educativa. Barcelona: Ediciones Experiencia, S. L.

Ley N ${ }^{\circ}$ 20.911. Diario Oficial de la República de Chile, Santiago, Chile, 28 de marzo de 2016.

Magendzo, A., \& Gazmiri, R. (2018). La educación del ciudadano. Pensar un currículum para la vida democrática. En A. Arratia \& L. Osandón (Eds.), Políticas para el desarrollo del currículum. Reflexiones y propuestas (pp. 247-269). Santiago: Unesco.

Pagés, J., \& Santisteban, A. (2010). La educación para la ciudadanía y la enseñanza de las ciencias sociales, la geografía y la historia. Íber, 64(2), 8-18.

Rodríguez, G., Gil, J., \& García, E. (1999). Metodología de la investigación cualitativa. Málaga: Ediciones Algibe.

Santisteban, A. (2009). Cómo trabajar en clase la competencia social y ciudadana. Aula de Innovación Educativa, 187, 12-15.

Stake, R. E. (2007). Investigación con estudio de casos ( $4^{\mathrm{a}}$ Ed.). Madrid: Morata.

Travers, M. (1986). Introducción a la investigación educacional. Barcelona: Paidos.

Villalón, G. (2012). Las representaciones sociales y las perspectivas prácticas sobre la ciudadanía de los profesores y estudiantes de profesorado en Historia, Geografía y Ciencias Sociales en Chile. En N. de Alba, García, F. García, A. \& Santisteban (Coords.), Educar para la participación ciudadana en la enseñanza de las Ciencias Sociales (pp. 303-311). Sevilla: Díada Editora.

Villalta, M., \& Ortega, M. (2019). Investigación-acción cooperativa: aprendiendo de una experiencia fallida. Persona, 1(22), 67-80.

Wood, P., \& Smith, J. (2018). Investigar en educación. Conceptos básicos y metodología para desarrollar proyectos de investigación. Madrid: Narcea. 